\title{
Institut für Keramik, Glas- und Baustofftechnik stellt sich neu auf
}

\author{
Aus dem bisherigen Institut für Keramik, Glas- und Baustofftechnik der TU Bergakademie \\ Freiberg wurden mit Wirkung zum 1. Oktober zwei neue Institute gegründet - das Institut für \\ Keramik, Feuerfest- und Verbundwerkstoffe sowie das Institut für Glas und Glastechnologie.
}

Am neuen Institut für Keramik, Feuerfestund Verbundwerkstoffe (IKFVW) der TU Bergakademie Freiberg (Bild 1) werden die ebenfalls neuen Professuren für Keramik, Feuerfest und metallokeramische Verbundwerkstoffe mit Prof. Christos Aneziris sowie für Bauchemie und Bauverbundwerkstoffe mit Prof. Thomas Bier zusammengefasst. So soll die inhaltliche Ausrichtung der beiden Bereiche in Forschung und Lehre deutlich gemacht werden.

Prof. Dr. Christos Aneziris, Leiter des neuen Instituts, möchte mit der Neustrukturierung außerdem die Sichtbarkeit der Keramik, Feuerfest- und Verbundwerkstoffe sowie der Baustoffchemie als fachübergreifende Disziplinen stärken. Am neuen Institut für Glas und Glastechnologie (IGT) werden die Professur für Glas- und Emailtechnik (aktuell vertreten durch Dr. Martin Kilo) sowie die Juniorprofessur für Energie- und Rohstoffeffiziente Glastechnologie (Jun.Prof. Sindy Fuhrmann) angesiedelt.

Durch das Institut wird die Glasforschung und -lehre an der TU Bergakademie Freiberg hervorgehoben und ihr im nationalen sowie im internationalen Kon-

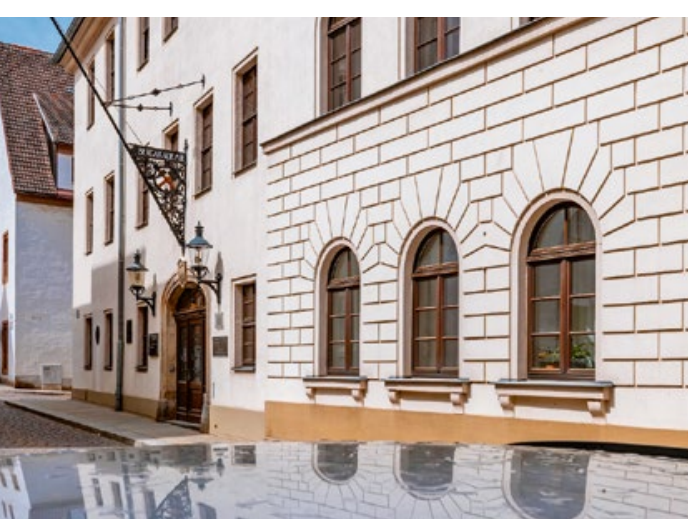

Bild 1 Das Hauptgebäude der TU Bergakademie Freiberg (@ TU Bergakademie Freiberg / D. Müller)

\section{Professor Aneziris erhält Auszeichnung der Deutschen Gesellschaft für Materialkunde}

Prof. Dr.-Ing. habil. Christos G. Aneziris (Bild 2) wurde im September mit der renommierten Tammann-Gedenkmünze geehrt. Die Deutsche Gesellschaft für Materialkunde (DGM) zeichnet damit die "weitreichenden wissenschaftlichen Arbeiten“ des Freiberger Keramik-Professors aus. „Dieses forschungs- und technologiebasierte Konzept ist die Grundlage seines weltweit anerkannten Wirkens als Hochschullehrer, Forscher und Partner der Industrie", so Prof. Dr. Peter Quirmbach von der Universität Koblenz-Landau in seiner Laudatio auf den Preisträger.

Professor Christos Aneziris ist seit 2001 Universitätsprofessor am Lehrstuhl für Keramik der TU Bergakademie Freiberg. Daneben engagiert sich der Experte für moderne feuerfeste Werkstoffe im Vorstand der Organisation Federation for International Refractory Research and Education (Kanada), ist Gastprofessor am Zhengzhou Institute of Aeronautical Industry Management, Henan (China), und Honorarprofessor an der Wuhan University of Science and Technology, Hubei (China). Für sein wissenschaftliches Wirken wurde Professor Aneziris bereits mit international angesehenen Auszeichnungen, wie beispielsweise dem Alf-

text als Institute of Glass Science and Technology eine neue Wirkung verliehen. Außerdem möchten die Professoren die Zusammenarbeit mit dem Glascampus Torgau als Netzwerkverbund und fachspezifischer Berufsbildungsstätte vorantreiben und die wissenschaftlich-technische

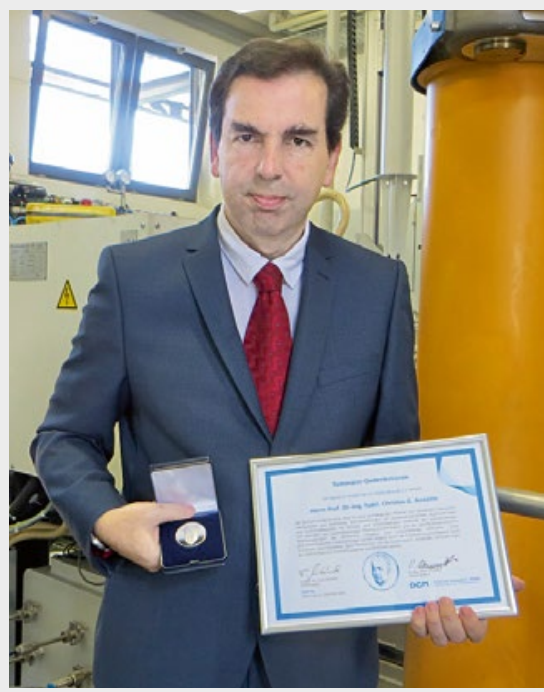

Bild 2 Prof. Dr. Christos Aneziris erhielt am 21. September die Tammann-Gedenkmünze 2020 der DGM. (๑ TU Bergakademie Freiberg)

red W. Allen Award der American Ceramic Society und dem Fellowship der European Ceramic Society ausgezeichnet.

"Ich freue mich sehr über die Anerkennung durch das Preiskuratorium und bedanke mich beim Vorstand der DGM für die Verleihung der Tammann-Gedenkmünze", sagte Prof. Dr. Christos Aneziris anlässlich der Online-Preisverleihung.

Vernetzung mit der nationalen wie auch internationalen Glasindustrie vertiefen. Leiter des neuen Instituts für Glas und Glastechnologie ist Dr. Kilo.

Kontakt:

TU Bergakademie Freiberg, Freiberg, www.tu-freiberg.de 